\title{
Estradiol valerate and dienogest: a new approach to oral contraception
}

This article was published in the following Dove Press journal:

International Journal of Women's Health

17 August 2011

Number of times this article has been viewed

\section{Jessica W Kiley}

Lee P Shulman

Section of Family Planning and Contraception, Department of Obstetrics and Gynecology, Feinberg School of Medicine of Northwestern University, Chicago, IL, USA
Correspondence: Jessica W Kiley 680 N Lake Shore Drive, Suite 1015, Chicago, IL 606II, USA

Tel +I 3 I2 4724683

Fax + I 31269587 II

Email jkiley@nmff.org

\begin{abstract}
Most combination oral contraceptives contain ethinyl estradiol and a progestin. A new and novel oral contraceptive formulation combines estradiol valerate (E2V) with dienogest (DNG) in a four-phase dosing regimen. $17 \beta$-estradiol is a naturally-occurring estrogen, and a contraceptive pill containing such an estrogen offers potential benefits with regard to metabolic side effects and adverse events. Dienogest is derived from 19-nortestosterone and exerts profound progestational effects on the endometrium, but it differs from other progestins in its class by its antiandrogenic activity. Estradiol valerate plus dienogest (E2V/DNG) is now available in a four-phasic regimen that integrates an estrogen stepdown and progestin stepup dosing approach along with a short two-day hormone-free interval. This regimen offers safe, reliable contraception and has been shown to be an effective treatment for heavy menstrual bleeding. Metabolic effects and adverse events appear similar to those reported with oral contraceptives containing ethinyl estradiol.
\end{abstract}

Keywords: estradiol valerate, dienogest, oral contraception, combination

\section{Introduction}

Combination oral contraceptive pills are the most commonly used form of reversible birth control in the US, with nearly 11 million women using them as their contraceptive method of choice. ${ }^{1}$ The majority of available combined oral contraceptive pills contain ethinyl estradiol as the estrogen component. Ethinyl estradiol is a synthetic hormone that efficiently delivers estrogen orally and is highly potent. Historically, there has been long-standing interest in developing a combined oral contraceptive pill containing estradiol, the most potent of the naturally occurring estrogens. The ability to do so has been thwarted by problems of poor oral absorption of estradiol and high rates of breakthrough bleeding. As such, a contraceptive pill regimen delivering estradiol instead of ethinyl estradiol was not successfully developed and, until recently, unavailable. ${ }^{2}$

Estradiol valerate + dienogest $(\mathrm{E} 2 \mathrm{~V} / \mathrm{DNG})$ is a new oral contraceptive formulation that provides effective contraception and satisfactory cycle control. Its novel regimen combines estradiol valerate $(\mathrm{E} 2 \mathrm{~V})$, which is a prodrug that delivers estradiol, with the progestin, dienogest (DNG), in a four-phasic dosing scheme designed to inhibit ovulation and minimize breakthrough bleeding. The 28-day formulation administers E2V $3 \mathrm{mg}$ on days 1 and 2, E2V $2 \mathrm{mg} / \mathrm{DNG} 2 \mathrm{mg}$ on days 3-7, E2V $2 \mathrm{mg} / \mathrm{DNG} 3 \mathrm{mg}$ on days 8-24, E2V $1 \mathrm{mg}$ on days 25 and 26, and placebo on days 27 and 28 (Figure 1). This new and unique regimen offers highly effective contraception, satisfactory cycle control, and has been shown to be an effective treatment for heavy menstrual bleeding. 


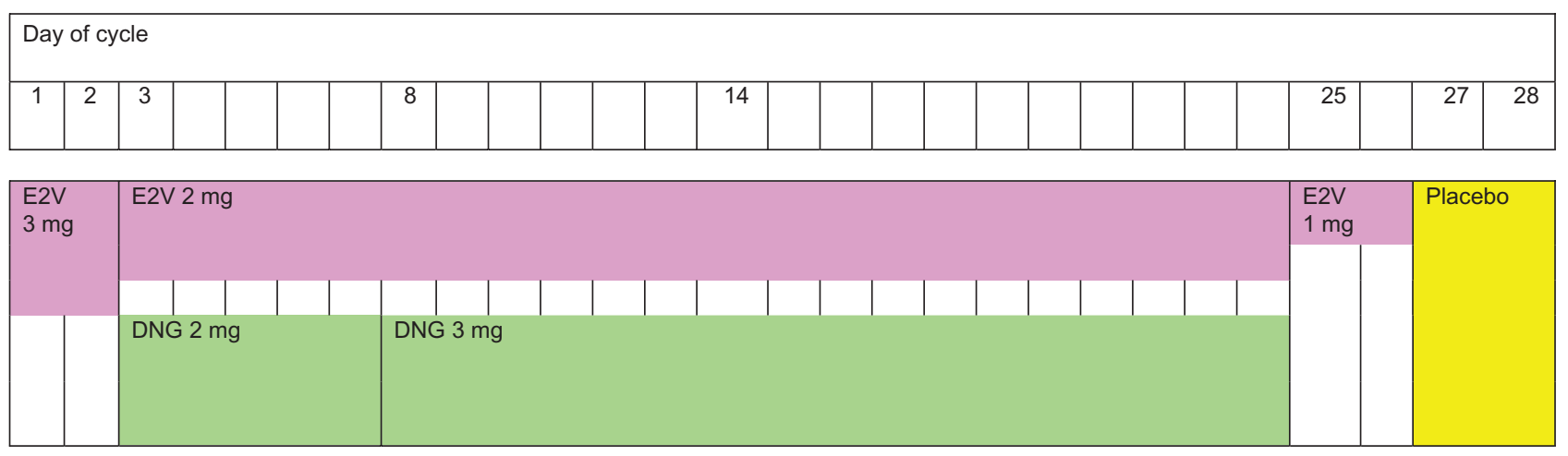

Figure I Four-phase dosing regimen of estradiol valerate (E2V)/dienogest (DNG).

\section{Pharmacology}

Interest in developing a combined oral contraceptive pill containing estradiol rather than ethinyl estradiol began years ago. These efforts were based on the premise that estradiol, a naturally occurring estrogen, would be associated with fewer side effects and improved tolerability. Upon oral administration, $\mathrm{E} 2 \mathrm{~V}$ is a prodrug in which the valerate side chain of the molecule is rapidly cleaved to form $17 \beta$-estradiol and valeric acid, with the estradiol being further metabolized to estrone and estrone sulfate. One milligram of $\mathrm{E} 2 \mathrm{~V}$ is equivalent to $0.76 \mathrm{mg}$ of $17 \beta$-estradiol. ${ }^{3}$ The biological effects of a $2 \mathrm{mg}$ daily dose of $\mathrm{E} 2 \mathrm{~V}$ is similar to that of ethinyl estradiol $20 \mu \mathrm{g}$ with regard to its effect on the hypothalamic-pituitary-ovarian axis and resulting effects on the ovaries and endometrium. However, a direct clinical comparison is not possible, owing to differences in the metabolism of the two estrogenic compounds. ${ }^{4}$

DNG is a progestin derived from 19-nortestosterone but is different in structure from other progestins in this class. The $17 \alpha$-ethinyl group, typical of many 19 -nortestoerone progestin derivatives, is replaced by a $17 \alpha$-cyanomethyl group in DNG. ${ }^{5}$ Similar to the other agents in its class, DNG exerts a strong progestational effect on the endometrium. However, unlike other 19-nortestosterone derivatives, it is characterized by an overall antiandrogenic hormonal milieu.

The novel four-phasic regimen was developed to provide for effective inhibition of ovulation and to minimize the breakthrough bleeding evident with earlier iterations of $17 \beta$ estradiol-containing and E2 V-containing regimens. The dosing schedule provides for an estrogen stepdown simultaneously with progestin stepup and a short, two-day, hormone-free interval. The first E2V/DNG oral contraceptive regimen in the US was recently approved by the Food and Drug Administration in 2010, under the brand name Natazia ${ }^{\circledR}$ (Bayer, Berlin, Germany), and the same regimen was approved earlier in Europe under the brand name Qlaira ${ }^{\circledR}$ (Bayer).
The rationale for this four-phasic regimen based on an estrogen stepdown and progestin stepup is that early estrogen dominance would confer endometrial proliferation in the first half of the cycle and prepare the endometrium for a midcyclic progestin effect. Use of increasing DNG dosages during the cycle would provide stability to the endometrial stroma, especially in the middle and end stages of the cycle.

\section{Mechanism of action}

Oral contraceptives containing ethinyl estradiol plus progestin provide for highly effective and reversible contraception. First-year perfect-use failure rates with the combined oral contraceptive pill are established as $0.3 \%$, with typical use rates being $8 \% .{ }^{6}$ The contraceptive action of the combined oral contraceptive pill results primarily from the actions of the hormones on the hypothalamus, pituitary, and ovaries. While combined oral contraceptive pills likely prevent pregnancy through several disparate mechanisms, their primary mechanism is the suppression of ovulation. The progestin component prevents the luteinizing hormone surge required for release of the ovum. It also serves to thicken cervical mucus and decrease tubal motility, creating a less facile passage for sperm. In addition, the progestin acts to thin the endometrium, resulting in tissue less receptive to implantation. The estrogen component of the pill serves to promote an acceptable cycle control and bleeding profile by stabilizing the endometrium during the days of exogenous estrogen ingestion. In addition, the estrogenic component also serves to augment contraceptive efficacy by inhibiting the release of follicle-stimulating hormone from the pituitary, which further inhibits the development of the dominant follicle. This, in turn, potentiates inhibition of the luteinizing hormone surge by progestin, without which ovulation and pregnancy cannot occur. ${ }^{7,8}$ 


\section{Efficacy}

Similar to other combined oral contraceptive pills, E2V/DNG provides effective birth control. Previously, several studies of pills containing estradiol consistently demonstrated adequate contraceptive efficacy, ${ }^{9-13}$ but issues of poor cycle control and unfavorable bleeding profiles led to high user dissatisfaction and unacceptable discontinuation rates in clinical trials, and prevented their entry into the contraceptive marketplace. ${ }^{3}$

Two studies have evaluated inhibition of ovulation with E2V/DNG. ${ }^{14}$ In these dose-finding studies, four different dosing regimens of E2V plus DNG were assessed. All four regimens involved E2V stepdown and DNG stepup, but the doses and length of exposure of each dose varied. Ovulation was assessed by transvaginal ultrasound measurement of ovarian follicle size and serum measurement of estradiol and progesterone levels, and the degree of ovulation was classified using Hoogland scores. Participants on each of the four regimens were followed for three consecutive cycles. These studies determined the lowest effective dose needed for efficacy, ie, E2V $3 \mathrm{mg}$ for two days, E2V $2 \mathrm{mg} / \mathrm{DNG}$ $2 \mathrm{mg}$ for five days, E2V $2 \mathrm{mg} / \mathrm{DNG} 3 \mathrm{mg}$ for 17 days, E2V $1 \mathrm{mg}$ for two days, then placebo for two days. No major safety concerns were identified with any of the regimens, and reported adverse events were similar to those occurring with use of any combined oral contraceptive pill.

A larger multicenter, open-label study evaluated healthy women on E2V/DNG over 20 cycles and documented low failure rates. ${ }^{3}$ Of 1377 women aged $18-50$ years receiving E2V/DNG, 13 pregnancies occurred, six of which were attributed to method failure (unadjusted Pearl Index 0.73; adjusted Pearl Index 0.34). In the subgroup of women aged 18-35 years, 12 pregnancies occurred, five of which were due to method failure (unadjusted Pearl Index 0.94; adjusted Pearl Index 0.40).

\section{Safety}

The main rationale for development of a combined oral contraceptive pill containing estradiol was based on the notion that a naturally occurring estrogen would be associated with fewer negative effects on lipid and glucose metabolism and hemostasis, and that adverse events would be minimized. In addition, a shorter half-life estrogenic component (estradiol) would possibly result in a lower rate of estrogen-associated complications, such as thromboembolic events, than those observed with the oral use of an estrogen (ethinyl estradiol) characterized by a longer half-life. The available literature suggests that the adverse events and metabolic effects of E2V/DNG are comparable with those of other combined oral contraceptive pills. Generally, adverse events in the reported studies were described as mild, and include headache, menstrual abnormality, and breast discomfort, similar to that reported with more conventional combined oral contraceptive pill regimens. ${ }^{2,15}$ Also similar to other combined oral contraceptive pills, serious adverse events were uncommon among women participating in the E2V/DNG trials. ${ }^{2}$ Of note is that the currently published studies of $\mathrm{E} 2 \mathrm{~V} / \mathrm{DNG}$ are too small to assess the frequency of venous thromboembolic events adequately, and other rare and uncommon adverse clinical outcomes in users of E2V/DNG. As such, while there is no current evidence to suggest an increased risk of thromboembolic events with E2V/DNG use compared with other estrogen-containing contraceptive methods, clinicians should consider the risk with this regimen to be similar to that of contraceptive regimens containing ethinyl estradiol and avoid using E2V/DNG in women who should not use estrogencontaining contraceptives.

In the multicenter Phase III efficacy trial described above, 917 of 1377 (66\%) participants reported one or more adverse events, with the vast majority of events being mild in nature. The most commonly reported adverse events included headache, diarrhea, and nasopharyngitis. Less common side effects, reported by $1 \%-3 \%$ of women, included breast pain and discomfort, acne, metrorrhagia, and weight gain. Serious adverse events were rare; those deemed possibly related to study medication included one case each of myocardial infarction, deep vein thrombosis, focal nodular hyperplasia of the liver, uterine leiomyoma, and ocular histoplasmosis syndrome. Again, this study was not powered to assess adequately the frequency of adverse events, such as thromboembolic events and other rare adverse events. Until further studies are done, clinicians should consider the safety profile with E2V/DNG to be similar to that of combined oral contraceptive pill regimens containing $35 \mu \mathrm{g}$ ethinyl estradiol or less.

Despite the current lack of robust comparative epidemiologic safety data, other studies have shown that the impact of E2V/DNG on lipid metabolism and hemostasis is apparently minimal. One open-label randomized study compared women on four-phasic E2V/DNG with those on triphasic ethinyl estradiol + levonorgestrel (ethinyl estradiol $0.03 \mathrm{mg} /$ levonorgestrel $0.05 \mathrm{mg}$ for six days, ethinyl estradiol $0.04 \mathrm{mg} /$ levonorgestrel $0.075 \mathrm{mg}$ for five days, ethinyl estradiol $0.03 \mathrm{mg} /$ levonorgestrel $0.125 \mathrm{mg}$ for 10 days, then placebo for seven days). ${ }^{16}$ Healthy participants aged 18-50 years were followed over seven cycles. Results suggested favorable effects on high-density lipoprotein levels in 
participants using E2V/DNG; high-density lipoprotein levels increased $7.9 \% \pm 21.8 \%$ in E2V/DNG users, compared with a $2.3 \% \pm 14.4 \%$ decrease in ethinyl estradiol/levonorgestrel users $(P=0.055)$. No significant differences in low-density lipoprotein levels, hemostatic characteristics, or carbohydrate metabolism were observed between the groups. Other studies measuring values for hemostasis parameters showed no clinically relevant change in most coagulation factors, including factors VII and VIII, antithrombin III, proteins C and $\mathrm{S}$, or in activated protein $\mathrm{C}$ resistance and plasminogen activator inhibitor activity. ${ }^{5}$ Comparisons of D-dimer and fibrinogen levels among E2V/DNG and ethinyl estradiol/ levonorgestrel users demonstrated little or no negative impact with either combined oral contraceptive pill formulation, with no clinically relevant differences between study groups. ${ }^{17}$ While these studies provide important information concerning the physiologic impact of E2V/DNG, they only provide information on a set of surrogate markers for which a clinical correlation with one or several adverse outcomes, including thromboembolic events, cannot and should not be made. Any consideration of a decreased or increased risk for thromboembolic events and other adverse outcomes must be directly determined by rigorous epidemiologic studies of E2V/DNG users.

These metabolic effects and their implications for clinical use have not been studied in women with medical comorbidities. Of interest is use of E2V/DNG in women with diabetes, obesity, and other medical conditions. At this time, contraindications to use of $2 \mathrm{~V} / \mathrm{DNG}$ are the same as those for ethinyl estradiol-containing combined oral contraceptive pills, including pregnancy, uncontrolled hypertension, migraine with aura, acquired or inherited predisposition to arterial or venous thrombosis, coronary artery disease, breast cancer, heavy smoking, complicated valvular heart disease, severe hepatic disease including liver cancer, and history of current stroke or thromboembolism.

\section{Bleeding profile}

Historically, there was interest in creating a "natural" or endogenous estradiol-based combined oral contraceptive pill, but problems with breakthrough bleeding in the first several regimens evaluated demonstrated considerable user dissatisfaction and high discontinuation rates, thus preventing the introduction of any of these regimens. Pharmacokinetic and dose-ranging studies eventually led to the current marketed combination of E2V/DNG in a regimen that provides for contraceptive effectiveness in addition to a well tolerated bleeding profile.
In a multicenter, randomized, and double-blind trial, ${ }^{18}$ 804 women aged 18-50 years were randomized to either four-phasic E2V/DNG or monophasic ethinyl estradiol $0.02 \mathrm{mg}$ /levonorgestrel $0.1 \mathrm{mg}$ in a standard 21/7 day regimen (ethinyl estradiol/levonorgestrel for 21 days, followed by placebo for seven days). In each of two 90-day reference periods, the mean number of bleeding and spotting days was less in the E2V/ DNG group than in the ethinyl estradiol/levonorgestrel group. In the first 90 days, the mean number was $17.3 \pm 10.4$ days for E2V/DNG users, and 21.5 \pm 8.6 days for ethinyl estradiol/ levonorgestrel $(P<0.0001)$. In the second 90 days, the mean number was $13.4 \pm 9.3$ days and $15.9 \pm 7.1$ days, respectively $(P<0.0001)$. Scheduled withdrawal bleeding episodes were shorter and lighter in participants treated with E2V/DNG than in those treated with ethinyl estradiol/levonorgestrel, with a mean length of withdrawal bleeding of 4.1-4.7 days with E2V/DNG, compared with 5.0-5.2 with ethinyl estradiol/ levonorgestrel $(P<0.05$ per cycle). In addition, the mean number of bleeding/spotting episodes was significantly less in the E2V/DNG group, with $3.7 \pm 1.4$ episodes, compared with $4.1 \pm 0.9$ episodes in the ethinyl estradiol/levonorgestrel group $(P<0.0001)$ in the first reference period, and $3.0 \pm 1.3$ versus $3.1 \pm 0.9$ episodes $(P=0.043)$ in the second reference period. However, it is important to note that the median numbers of bleeding episodes were identical between groups in both periods, in that there were 4.0 episodes in the first 90 days and 3.0 episodes in the second 90 days, so the clinical relevance of the statistically significant findings is questionable. Furthermore, the clinical relevance of the fewer total number of bleeding/ spotting days is unclear. With regard to cycle control, most women in both groups experienced scheduled withdrawal bleeding, but the absence of scheduled withdrawal bleeding occurred more often in E2V/DNG users. The mean percentage of women on E2V/DNG who had an absent withdrawal bleeding episode was $19.4 \%$ over seven cycles, compared with $7.7 \%$ of women using ethinyl estradiol/levonorgestrel $(P<0.0001$ for each cycle), and most of these women experienced a single absent withdrawal bleeding episode. An absence of any bleeding, scheduled or unscheduled, occurred in $15.4 \%$ of cycles with E2V/DNG and $4.5 \%$ of cycles with ethinyl estradiol/ levonorgestrel. Rates of intermenstrual bleeding were similar between groups; approximately $14 \%$ and $12 \%$ of E2V/DNG and ethinyl estradiol/levonorgestrel users, respectively, experienced such bleeding. Typically the bleeding was reported to be light, and occurred most commonly during the first treatment cycle. Patient satisfaction in the two groups was similar, with most participants $(79 \%)$ reporting being satisfied with their treatment regimen. 


\section{Patient satisfaction}

Consistent and correct use of prescribed contraceptive methods depends on several different and unrelated factors, but satisfaction with a chosen method is a major determinant of successful and ongoing use. ${ }^{19}$ As described above, use of E2V/DNG is generally well tolerated. In the large Phase III efficacy trial, ${ }^{3} 140$ of 1377 participants (10.2\%) discontinued the study medication due to adverse events, with menstrual irregularity $(1.7 \%)$, acne $(1.0 \%)$, and weight gain $(0.9 \%)$ being the most commonly cited reasons for discontinuation. In the trial comparing bleeding profiles and cycle control with E2V/DNG and ethinyl estradiol/levonorgestrel, ${ }^{18}$ a subjective assessment of satisfaction was obtained. Participants were queried regarding their satisfaction with treatment and asked to rate their level of satisfaction according to five categories, ie, very satisfied, somewhat satisfied, neither satisfied nor dissatisfied, dissatisfied, or very dissatisfied. Most women in both groups reported that they were somewhat satisfied or very satisfied with treatment $(79.4 \%$ in the E2V/DNG group, $79.9 \%$ in the ethinyl estradiol/levonorgestrel group). Over one third of women in both groups rated themselves as very satisfied with treatment (39.8\% in the E2V/DNG group, $35.3 \%$ in the ethinyl estradiol/levonorgestrel group).

\section{Noncontraceptive benefits}

Aside from providing reliable pregnancy prevention, combined oral contraceptive pills offer certain gynecologic and nongynecologic health benefits. These noncontraceptive benefits include menstrual cycle control, decreased menstrual blood loss, reduced dysmenorrhea, prevention of ovarian cyst formation, decreased ectopic pregnancy risk, protection against endometrial, ovarian and colon cancer, decreased risk of pelvic inflammatory disease, less benign breast disease, treatment of acne, and increased bone mass. ${ }^{20}$

Specifically, heavy menstrual bleeding (previously described as "menorrhagia") is a common indication for prescription of the combined oral contraceptive pill, and E2V/DNG has been shown to treat heavy menstrual bleeding effectively. Two randomized, placebo-controlled studies have assessed the effects of E2V/DNG on heavy menstrual bleeding. In the first trial, 190 women with heavy or prolonged menstrual bleeding were randomized in a 2:1 fashion to E2V/DNG and placebo. Participants using E2V/DNG experienced significantly higher response rates than those on placebo ( $43.75 \%$ versus $4.17 \%, P<0.0001$ ). Quantitative measurement of menstrual blood loss showed that E2V/DNG users experienced greater reductions in blood loss compared with placebo $(-353.1$ versus $-130.4 \mathrm{~mL}, P<0.0001)$.
Correspondingly, hemoglobin, hematocrit, and ferritin levels in E2V/DNG users were significantly improved. Efficacy for treatment of heavy and/or prolonged bleeding was similarly demonstrated in the second trial, ${ }^{21}$ in which participants receiving E2V/DNG experienced significantly less menstrual blood loss than those using placebo (adjusted mean difference $-373 \mathrm{~mL}, 95 \%$ confidence interval $-490,-255$; $P<0.0001)$. Increased hemoglobin and ferritin concentrations were similarly found in this trial. These trials provide compelling evidence that E2V/DNG offers effective treatment for heavy menstrual bleeding.

Despite the lack of a study directly comparing the effects of E2V/DNG and an ethinyl estradiol-containing combined oral contraceptive pill on heavy menstrual bleeding, the aforementioned trials were used by European Union regulators to endorse the indication of E2V/DNG for the treatment of heavy menstrual bleeding in women without organic pathology who choose to use an oral contraceptive. In the US, the $\mathrm{E} 2 \mathrm{~V} / \mathrm{DNG}$ regimen is indicated solely for the prevention of pregnancy. Additional study is needed to evaluate the clinical advantages of E2V/DNG over traditional formulations. Direct comparison of magnitude of reduced menstrual blood loss in women with menorrhagia is required, because it is well documented that ethinyl estradiol-containing pills confer such an effect. ${ }^{7,8}$ Furthermore, issues of cost and access must be considered when appraising a new medication. The potential clinical benefits of $\mathrm{E} 2 \mathrm{~V} / \mathrm{DNG}$ use must take into account its relative expense and, in turn, restricted availability, especially in resource-poor settings.

\section{Conclusion}

The new combined E2V/DNG oral contraceptive pill regimen offers reliable contraceptive efficacy, similar to that observed and expected with all combined oral contraceptive pills. Effects on carbohydrate and lipid metabolism and hemostasis factors are most likely minimal and not likely to affect women in a healthy population of users adversely. Menstrual cycle control and bleeding profiles have been reported to be well accepted by study participants, and most users in trials report satisfaction with the method. Frequency and type of adverse events are similar to those reported by users of ethinyl estradiol-containing combined oral contraceptive pills, and are not typically severe. However, current evidence supports the premise that the safety profile of E2V/DNG is similar to that of low-dose combined oral contraceptive pills that contain ethinyl estradiol, and thus it should not be provided to women in whom estrogen-containing contraceptive use is contraindicated. 
E2V/DNG has also been demonstrated to be effective for the treatment of heavy menstrual bleeding in women choosing to use the regimen to prevent pregnancy. Despite data demonstrating similar cycle control between E2V/DNG and older formulations, the current literature lacks comparative trials of E2V/DNG and ethinyl estradiol-containing pills with regard to heavy menstrual bleeding. As with any new combined oral contraceptive pill formulation, additional and more extensive data regarding safety, effectiveness, and side effects will need to be elicited from its ongoing use. Nonetheless, the current evidence supports the use of E2V/DNG as a first-line, mainstream oral contraceptive option for women seeking effective, reversible, and well tolerated oral contraception.

\section{Disclosure}

LPS is a consultant and provides educational programs for Bayer Pharmaceuticals. JWK has no conflict of interest to report in this work.

\section{References}

1. Mosher WD, Jones J. Use of contraception in the United States: 1982-2008. Vital Health Stat 23. 2010;29:1-44.

2. Jensen JT. Evaluation of a new estradiol oral contraceptive: Estradiol valerate and dienogest. Expert Opin Pharmacother. 2010;11(7): $1147-1157$.

3. Palacios S, Wildt L, Parke S, Machlitt A, Romer T, Bitzer J. Efficacy and safety of a novel oral contraceptive based on oestradiol (oestradiol valerate/dienogest): A Phase III trial. Eur J Obstet Gynecol Reprod Biol. 2010;149(1):57-62.

4. Natazia - a new oral contraceptive. Med Lett Drugs Ther. 2010;52(1346): $71-72$.

5. Hoy SM, Scott LJ. Estradiol valerate/dienogest: In oral contraception. Drugs. 2009;69(12):1635-1646.

6. Trussell J. Contraceptive failure in the United States. Contraception. 2004;70(2):89-96.

7. Speroff L, Darney PD. Oral contraception. In: Speroff L, Darney PD, editors. A Clinical Guide for Contraception. Philadelphia, PA: Lippincott Williams and Wilkins; 2011.

8. Kiley J, Hammond C. Combined oral contraceptives: A comprehensive review. Clin Obstet Gynecol. 2007;50(4):868-877.
9. World Health Organization Task Force on Oral Contraception. A randomized, double-blind study of two combined oral contraceptives containing the same progestogen, but different estrogens. Contraception. 1980;21(5):445-459.

10. Astedt B, Jeppsson S, Liedholm P, Rannevik G, Svanberg L. Clinical trial of a new oral contraceptive pill containing the natural oestrogen 17 beta-oestradiol. Br J Obstet Gynaecol. 1979;86(9):732-736.

11. Csemiczky G, Dieben T, Coeling Bennink HJ, Landgren BM. The pharmacodynamic effects of an oral contraceptive containing $3 \mathrm{mg}$ micronized 17 beta-estradiol and $0.150 \mathrm{mg}$ desogestrel for 21 days, followed by $0.030 \mathrm{mg}$ desogestrel only for 7 days. Contraception. 1996; 54(6):333-338.

12. Hirvonen E, Allonen H, Anttila M, et al. Oral contraceptive containing natural estradiol for premenopausal women. Maturitas. 1995;21(1): $27-32$.

13. Serup J, Bostofte E, Larsen S, Westergaard J, Lebech PE. Natural oestrogens for oral contraception. Lancet. 1979;2(8140):471-472.

14. Endrikat J, Parke S, Trummer D, Schmidt W, Duijkers I, Klipping C. Ovulation inhibition with four variations of a four-phasic estradiol valerate/dienogest combined oral contraceptive: Results of two prospective, randomized, open-label studies. Contraception. 2008;78(3):218-225.

15. Zeun S, Lu M, Uddin A, Zeiler B, Morrison D, Blode H. Pharmacokinetics of an oral contraceptive containing oestradiol valerate and dienogest. Eur J Contracept Reprod Health Care. 2009;14(3):221-232.

16. Parke S, Nahum G, Mellinger U, Junge W. Metabolic effect of a new four-phasic oral contraceptive containing estradiol valerate and dienogest. Obstet Gynecol. 2008;111 Suppl 4:S12-S13.

17. Parke S, Junge W, Mellinger U, Duijkers I, Klipping C. Comparative effects of a four-phasic regimen of estradiol valerate/dienogest versus ethinylestradiol/levonorgestrel on haemostatic parameters. Hum Reprod. 2008;23 Suppl 1:i78-i79.

18. Ahrendt HJ, Makalova D, Parke S, Mellinger U, Mansour D. Bleeding pattern and cycle control with an estradiol-based oral contraceptive: A seven-cycle, randomized comparative trial of estradiol valerate/ dienogest and ethinyl estradiol/levonorgestrel. Contraception. 2009; 80(5):436-444.

19. Moreau C, Cleland K, Trussell J. Contraceptive discontinuation attributed to method dissatisfaction in the United States. Contraception. 2007;76(4):267-272.

20. Burkman R, Schlesselman JJ, Zieman M. Safety concerns and health benefits associated with oral contraception. Am J Obstet Gynecol. 2004;190 Suppl 4:S5-S22.

21. Fraser I, Zeun S, Machlitt A, Mellinger U. A novel oral contraceptive comprising estradiol valerate/dienogest for the treatment of heavy and/ or prolonged menstrual bleeding without organic cause: A double-blind, randomised, placebo-controlled trial. Int J Gynecol Obstet. 2009; 107 Suppl 2:S183.
International Journal of Women's Health

\section{Publish your work in this journal}

The International Journal of Women's Health is an international, peerreviewed open-access journal publishing original research, reports, reviews and commentaries on all aspects of women's healthcare including gynecology, obstetrics, and breast cancer. Subject areas include: Chronic conditions (migraine headaches, arthritis, osteoporosis);

\section{Dovepress}

Endocrine and autoimmune syndromes; Sexual and reproductive health; Psychological and psychosocial conditions. The manuscript management system is completely online and includes a very quick and fair peer-review system. Visit http://www.dovepress.com/ testimonials.php to read real quotes from published authors. 